\title{
Bone marrow aplasia in B cell chronic lymphocytic leukaemia: Successful treatment with antithymocyte globulin
}

\author{
R Singal, D A Winfield, $M$ Greaves
}

\begin{abstract}
Pure red cell aplasia is a rare but well known association of chronic lymphocytic leukaemia (CLL). Pancytopenia due to bone marrow aplasia has not been previously described in CLL. A 42 year old man with B cell CLL became severely pancytopenic with bone marrow aplasia. Bone marrow culture resulted in a greatly reduced colony formation. High dose corticosteroids and intravenous immunoglobulin treatment were unsuccessful. Prompt and complete marrow recovery ensued after administration of antithymocyte globulin.
\end{abstract}

Many patients with chronic lymphocytic leukaemia develop anaemia during the course of their disease. Some with end stage chronic lymphocytic leukaemia (CLL) become pancytopenic due to replacement of normal precursors by leukaemic cells. Pure red cell aplasia (PRCA) is said to develop in about $6 \%$ of cases of CLL and is thought to be immunologically mediated. ${ }^{1} \mathrm{~T}-\gamma$ cells in such patients may suppress erythroid progenitor cell growth. ${ }^{1}$ Antithymocyte globulin and cyclosporin-A have been successfully used in treatment. ${ }^{23}$

We present the first case report, to our knowledge, of pancytopenia caused by marrow aplasia complicating B-cell CLL. Furthermore, despite the failure of high dose corticosteroids and intravenous human IgG to influence the disorder, normal blood cell counts were rapidly achieved after administration of antithymocyte globulin.

Department of Haematology, Royal Hallamshire Hospital,

Glossop Road, Sheffield S10 2JF

R Singal

D A Winfield

M Greaves

Correspondence to: Dr Singal

Accepted for publication 4 April 1991

\section{Case report}

A 42 year old man presented to the eye casualty department in 1985 for "spots in his vision". Fundus examination revealed a left retinal haemorrhage which slowly resolved. A full blood count was normal. He sustained a similar episode in August 1988. A full blood count showed a haemoglobin concentration of $18.0 \mathrm{~g} / \mathrm{dl}$, a white cell count of $14.7 \times 10^{9} / 1$ with a lymphocytosis of $8.8 \times 10^{9} / 1$, and a platelet count of $189 \times 10^{9} / 1$. There were no systemic symptoms and physical examination was unremarkable. A diagnosis of "stress" polycythaemia and B-CLL was made in view of blood film appearances, normal isotopic red cell mass and reduced plasma volume, and an immunophenotype study showing a monoclonal B cell proliferation with positive CD5 and CD23 expression. The polycythaemia resolved after he gave up smoking and no treatment was offered for the CLL.

After 12 months the lymphocyte count was $25 \times 10^{9} / 1$ and his spleen was palpable below the costal margin (figure). Three months later he was aware of cervical and axillary lymphadenopathy and shortly after had symptoms of anaemia with a haemoglobin of $10 \cdot 1 \mathrm{~g} / \mathrm{dl}$, a white cell count of $109 \times 10^{9} / 1$, lymphocytes $99 \times 10^{9} / 1$, neutrophils $9.8 \times 10^{9} / 1$ and $a$ platelet count of $173 \times 10^{9} / 1$. Chlorambucil $5 \mathrm{mg}(60 \mu \mathrm{g} / \mathrm{kg})$ daily was administered.

He was admitted with severe anaemia two weeks after starting treatment (haemoglobin $4.6 \mathrm{~g} / \mathrm{dl}$ ). Bone marrow examination showed a severely hypercellular marrow diffusely infiltrated by a population of small lymphoid cells with no red cell precursors. Myeloid series and megakaryocytes were adequately represented. The direct antiglobulin test was positive (IgG + C3d) and serum haptoglobin undetectable. The anaemia was considered to be due to haemolysis and red cell hypoplasia, and treatment with high dose corticosteroids was introduced (prednisolone $1 \mathrm{mg} / \mathrm{kg} /$ day). After four weeks of Chlorambucil the lymphadenopathy resolved and splenomegaly became less pronounced. The neutrophil and platelet counts fell, however, and at seven weeks neutrophils were absent from peripheral blood and his platelet count reached $25 \times 10^{9} / 1$. A bone marrow trephine biopsy specimen was hypocellular with a lymphoid infiltrate and absence of megakaryocytes, myeloid, and erythroid precursors. Haematinic concentrations were normal and a Ham's test negative. Bone marrow culture showed reduced colony counts with three granulocyte-macrophage colonies per $10^{5}$ marrow cells (normal range 50-100 g-m colonies per $10^{5}$ marrow cells) and one erythroid colony per $10^{5}$ marrow cells (normal range $80-100$ ). 
Blood picture and white cell differential from presentation to 21 months. $S=$ splenomegaly; $L N=$ lymphadenopathy.

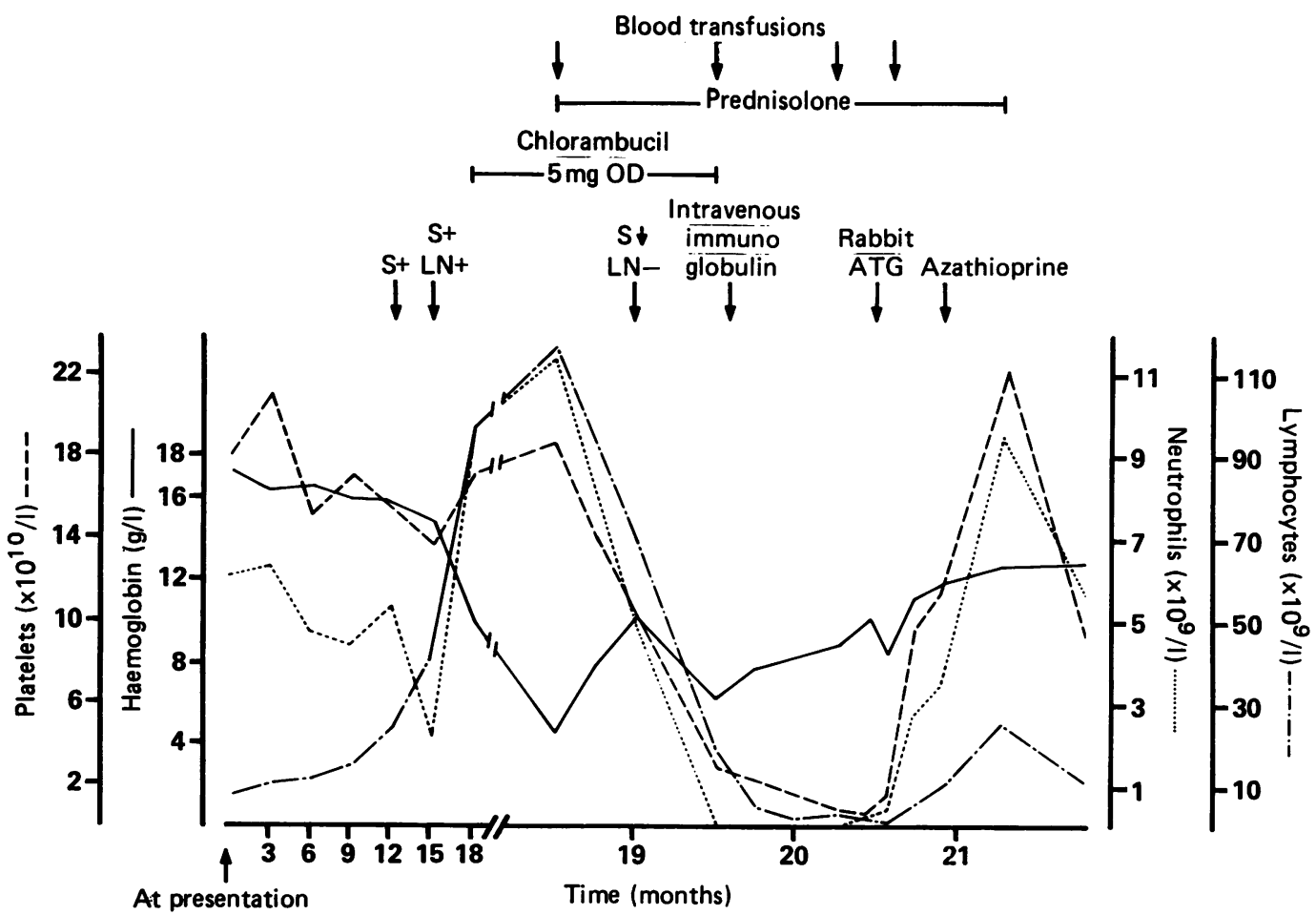

Chlorambucil was discontinued. During the pancytopenic phase he became feverish which responded to broad spectrum antibiotics, though the blood cultures were negative. Haemorrhagic manifestations were managed by platelet transfusions. Further red cell transfusions were administered (figure). A course of high dose human IgG (immunoglobulin $0.5 \mathrm{~g} / \mathrm{kg} / 1 \mathrm{~kg}$ for five days) failed to influence the progression of aplasia (platelet nadir $\left.4 \times 10^{9} / 1\right)$.

After four weeks of severe pancytopenia horse anti-lymphocyte globulin (Mérieux) (15 $\mathrm{mg} / \mathrm{kg}$ ) was administered, but the test dose caused a severe febrile reaction. Subsequently a course of rabbit anti-thymocyte globulin (Mérieux) $(2.5 \mathrm{mg} / \mathrm{kg}$ daily for five days) was successful. Within one week the neutrophil and platelet counts approached normal and reticulocytes $(0.8 \%)$ appeared for the first time in the peripheral blood. A direct antiglobulin test remained positive. Further transfusions were not necessary. Azathioprine $50 \mathrm{mg}$ three times a day was started and at the time of writing (six weeks after recovery) blood counts are normal, with a lymphocytosis of $8.16 \times 10^{9} / 1$, and film appearances consistent with CLL.

\section{Discussion}

Pure red cell aplasia is a rare but known association of chronic lymphocytic leukaemia. The incidence of PRCA is said to be about $6 \%$ and it is reported in both B-cell CLL and T-cell CLL. Rarely is it a presenting feature. ${ }^{1}$

Inhibition of erythropoiesis is thought to be immunologically mediated. Abnormal T cells with receptors for IgG ( $T-\gamma$ cells) have been found in such cases and these cells can inhibit the growth of marrow progenitor cells from normal subjects in vitro. ${ }^{14}$ Cyclosporin and antithymocyte globulin have both been used

The mechanism by which these drugs act is not understood. As both are immunosuppressive agents and are known to cause depletion of total $\mathrm{T}$ cells an effect on abnormal $\mathrm{T}-\gamma$ cells might contribute to their beneficial effect. ${ }^{23}$ Antithymocyte globulin also stimulates the immune system and has been shown in vitro to down-regulate the production of negative lymphokines and enhance the release of haemopoietins after interacting with bone marrow $T$ and non- $T$ cells from patients with severe aplastic anaemia. ${ }^{25}$

To our knowledge, marrow aplasia in CLL has not been previously described. The picture was transiently that of PRCA, as shown by bone marrow examination performed two weeks after starting Chlorambucil, but pancytopenia ensued in spite of resolution of lymphadenopathy and splenomegaly. A repeat bone marrow examination performed eight weeks after starting Chlorambucil showed a hypocellular marrow with infiltration by lymphoid cells and absence of megakaryocytes, myeloid, and erythroid precursors. This, together with reduced colony count on bone marrow culture, is consistent with bone marrow aplasia.

Chlorambucil in large doses could conceivably result in a similar picture, but a rapidly progressive anaemia followed the start of Chlorambucil treatment. More importantly, the dose given ( $60 \mu \mathrm{g} / \mathrm{kg}$ daily for six weeks) is considerably below the recommended dose for this drug given as a single agent $(100-200 \mu \mathrm{g} /$ $\mathrm{kg}$ daily for four to eight weeks) and would not be expected to produce profound pancytopenia. Furthermore, the very prompt and complete response to anti-thymocyte globulin is consistent with an immune mediated aplastic state. for treatment of PRCA in CLL. ${ }^{23}$ 
The early rapid fall in haemoglobin with reticulocytopenia and a positive DAT with undetectable serum haptoglobin is interesting. This was noted at a time when normal blood neutrophil and platelet counts were maintained and is consistent with an autoimmune pathogenesis, the antibody perhaps also being active against red cell precursors. The marrow appearances supported this possibility.

In summary, a severe anaemia developed in a patient with B-CLL at a time of increasing disease activity. Evidence for immune haemolytic anaemia was detected but the picture rapidly developed into that of bone marrow aplasia. A prompt and complete response to anti-thymocyte globulin was obtained, suggesting a role for $\mathrm{T}$ lymphocytes in the pathogenesis of this unique complication of CLL.
We are grateful to Dr N Testa (Department of Experimental Haematology, Christie Hospital, Manchester) for performing bone marrow culture.

1 Chikkapa G, Zarrabi MH, Tsan MF. Pure red cell aplasia in patients with chronic lymphocytic leukaemia. Medicine patients with chror

2 Radosevich CA, Gordon LI, Weil SC, Marden RJ, Rosen ST. Complete resolution of pure red cell aplasia in a patient with chronic lymphocytic leukaemia following antithymocyte globulin therapy. $J A M A$ 1988;259:723-5.

3 Christen R, Morant R, Fehr J. Cyclosporin A therapy of pure red cell aplasia in a patient with B-cell chronic lymphocytic leukaemia. Eur J Haematol 1989;42:303-7.

4 Mangan KF, Chikkappa G, Farley PC. T gamma cells suppress growth of erythroid colony forming units in vitro in the pure red cell aplasia of B-cell chronic lymphocytic leukaemia. J Clin Invest 1982;70:1148-56.

5 Tong J, Bacigalupo A, Piaggio G, Figari O, Marmont A Effect of antilymphocyte globulin (ALG) on bone marrow $\mathrm{T} /$ non- $\mathrm{T}$ cells from aplastic anaemia patients and normal T/non- $\mathrm{T}$ cells from aplastic anaemia patien

\title{
Bleeding diathesis coincident with chronic myelomonocytic leukaemia
}

\author{
S E Heard, P Revell, L J Holland, A D F Hurdle, G F Savidge
}

Supra-Regional Haemophilia Centre, St Thomas's Hospital, Lambeth Palace Road, London SE1 7EH S E Heard $P$ Revell L J Holland

G F Savidge

Epsom District Hospital

A D F Hurdle

Correspondence to: Dr S E Heard, 49 Fair-AFar, Edinburgh EI14 6QB. Accepted for publication 9 May 1991

\begin{abstract}
Two important haematological problems were found in an otherwise healthy 78 year old man: chronic myelomonocytic leukaemia; and a complex, acquired, hyperfibrinolytic bleeding disorder characterised by prolonged coagulation times, deficiency of coagulation factors $\mathrm{V}, \mathrm{X}$, and XI, anti-thrombin III and proteins $C$ and $S$, with high concentrations of circulating tissue plasminogen activator, and low concentrations of plasminogen activator inhibitor.

There may be a causal relation between the two conditions, with the peripheral blood monocytes mediating the hyperfibrinolytic process by the abnormal production of tissue plasminogen activator, though no previous description of a similar association has been reported.
\end{abstract}

\section{Case report}

Two years before referral, the coincidental diagnosis of a myeloproliferative disorder had been made during the investigation of haematuria in a 78 year old man. Urate bladder stones secondary to hyperuricaemia were diagnosed. The spleen was palpable to $3 \mathrm{~cm}$ and the blood count showed a haemoglobin $161 \mathrm{~g} / 1$, white cell count of $83.7 \times 10^{9} / 1$, and a platelet count of $417 \times 10^{9} / 1$. A bone marrow aspirate and trephine biopsy specimen showed increased cellularity with high reticulin and myeloid activity. The karyotype was normal and the leucocyte alkaline phosphatase score was increased. There was no history of major surgery.

One month later an elective prostatic biopsy was performed, leading to excessive bleeding. The results of a coagulation screen taken at the time are shown in the table. The bleeding stopped in response to fresh frozen plasma (FFP). The biopsy specimen was histologically normal.

The following year an inguinal hernia repair resulted in prolonged bleeding persisting for four weeks, requiring transfusion of red cells and daily FFP infusions. Coagulation results obtained at the time (1987) are shown in the table. An orchidectomy became necessary after the formation of a large scrotal haematoma, the histology of which was normal.

The initial referral to this centre (1988) was prior to dental treatment. The patient was otherwise well with no spontaneous bleeding or bruising and the myeloproliferative disorder was well controlled by intermittent oral hydroxyurea. Full blood count showed a haemoglobin of $137 \mathrm{~g} / \mathrm{l}$, a white cell count of $14.7 \times 10^{9} / 1$, with neutrophils $10.3 \times 10^{9} / 1$, lymphocytes $2.4 \times 10^{9} / 1$, monocytes $1.2 \times$ $10^{9} / 1$, and eosinophils $0.8 \times 10^{9} / 1$. Coagulation investigations were performed including an 